\title{
Determinants of Block Matrices
}

\author{
John R. Silvester
}

\section{Introduction}

Let us first consider the $2 \times 2$ matrices $\mathbf{M}=\left(\begin{array}{cc}a & b \\ c & d\end{array}\right)$ and $\mathbf{N}=\left(\begin{array}{ll}e & f \\ g & h\end{array}\right)$. Their sum and product are given by

$$
\mathbf{M}+\mathbf{N}=\left(\begin{array}{ll}
a+e & b+f \\
c+g & d+h
\end{array}\right) \quad \text { and } \quad \mathbf{M N}=\left(\begin{array}{cc}
a e+b g & a f+b h \\
c e+d g & c f+d h
\end{array}\right) .
$$

Here the entries $a, b, c, d, e, f, g, h$ can come from a field, such as the real numbers, or more generally from a ring, commutative or not. Indeed, if $F$ is a field, then the set $R={ }^{n} F^{n}$ of all $n \times n$ matrices over $F$ forms a ring (non-commutative if $n \geq 2$ ), because its elements can be added, subtracted and multiplied, and all the ring axioms (associativity, distributivity, etc.) hold. If $a, b, \ldots, h$ are taken from this ring $R$, then $\mathbf{M}, \mathbf{N}$ can be thought of either as members of ${ }^{2} R^{2}(2 \times 2$ matrices over $R)$ or as members of ${ }^{2 n} F^{2 n}$. It is well-known fact, which we leave the reader to investigate, that whether we compute with these matrices as $2 n \times 2 n$ matrices, or as $2 \times 2$ "block" matrices (where the blocks $a, b, \ldots$ are $n \times n$ matrices, i.e., elements of $R$ ) makes no difference as far as addition, subtraction and multiplication of matrices is concerned. (See for example [2], p. 4, or [6], pp. 100-106.) In symbols, the rings ${ }^{2} R^{2}$ and ${ }^{2 n} F^{2 n}$ can be treated as being identical: ${ }^{2} R^{2}={ }^{2 n} F^{2 n}$, or ${ }^{2}\left({ }^{n} F^{n}\right)^{2}={ }^{2 n} F^{2 n}$. More generally, we can partition any $m n \times m n$ matrix as an $m \times m$ matrix of $n \times n$ blocks: ${ }^{m}\left({ }^{n} F^{n}\right)^{m}={ }^{m n} F^{m n}$.

The main point of this article is to look at determinants of partitioned (or block) matrices. If $a, b, c, d$ lie in a ring $R$, then provided that $R$ is commutative there is a determinant for $\mathbf{M}$, which we shall write as $\operatorname{det}_{R}$, thus: $\operatorname{det}_{R} \mathbf{M}=a d-b c$, which of course lies in $R$. If $R$ is not commutative, then the elements $a d-b c, a d-c b, d a-b c$, $d a-c b$ may not be the same, and we do not then know which of them (if any) might be a suitable candidate for $\operatorname{det}_{R} \mathbf{M}$. This is exactly the situation if $R={ }^{n} F^{n}$, where $F$ is a field (or a commutative ring) and $n \geq 2$; so to avoid the difficulty we take $R$ to be, not the whole of the matrix ring ${ }^{n} F^{n}$, but some commutative subring $R \subset{ }^{n} F^{n}$. The usual theory of determinants then works quite happily in ${ }^{2} R^{2}$, or more generally in ${ }^{m} R^{m}$, and for $\mathbf{M} \in{ }^{m} R^{m}$ we can work out $\operatorname{det}_{R} \mathbf{M}$, which will be an element of $R$. But $R \subset{ }^{n} F^{n}$, so $\operatorname{det}_{R} \mathbf{M}$ is actually a matrix over $F$, and we can work out $\operatorname{det}_{F}\left(\operatorname{det}_{R} \mathbf{M}\right)$, which will be an element of $F$. On the other hand, since $R \subset{ }^{n} F^{n}$, we have $\mathbf{M} \in{ }^{m} R^{m} \subset{ }^{m}\left({ }^{n} F^{n}\right)^{m}={ }^{m n} F^{m n}$, so we can work out $\operatorname{det}_{F} \mathbf{M}$, which will also be an element of $F$. Our main conclusion is that these two calculations give the same result:

THEOREM 1. Let $R$ be a commutative subring of ${ }^{n} F^{n}$, where $F$ is a field (or a commutative ring), and let $\mathbf{M} \in{ }^{m} R^{m}$. Then

$$
\operatorname{det}_{F} \mathbf{M}=\operatorname{det}_{F}\left(\operatorname{det}_{R} \mathbf{M}\right) \text {. }
$$


For example, if $\mathbf{M}=\left(\begin{array}{ll}\mathbf{A} & \mathbf{B} \\ \mathbf{C} & \mathbf{D}\end{array}\right)$ where $\mathbf{A}, \mathbf{B}, \mathbf{C}, \mathbf{D}$ are $n \times n$ matrices over $F$ which all commute with each other, then Theorem 1 says

$$
\operatorname{det}_{F} \mathbf{M}=\operatorname{det}_{F}(\mathbf{A D}-\mathbf{B C}) .
$$

Theorem 1 will be proved later. First, in section 2 we shall restrict attention to the case $m=2$ and give some preliminary (and familiar) results about determinants of block diagonal and block triangular matrices which, as a by-product, yield a proof by block matrix techniques of the multiplicative property of determinants. In section 3 we shall prove something a little more general than Theorem 1 in the case $m=2$; and Theorem 1 itself, for general $m$, will be proved in section 4 .

\section{The multiplicative property}

Let $\mathbf{M}=\left(\begin{array}{ll}\mathbf{A} & \mathbf{B} \\ \mathbf{C} & \mathbf{D}\end{array}\right)$, where $\mathbf{A}, \mathbf{B}, \mathbf{C}, \mathbf{D} \in{ }^{n} F^{n}$, so that $\mathbf{M} \in{ }^{2 n} F^{2 n}$. As a first case, suppose $\mathbf{B}=\mathbf{C}=\mathbf{O}$, the $n \times n$ zero matrix, so that $\mathbf{M}=\left(\begin{array}{ll}\mathbf{A} & \mathbf{O} \\ \mathbf{O} & \mathbf{D}\end{array}\right)$, a block-diagonal matrix. It is a well-known fact that

$$
\operatorname{det}_{F}\left(\begin{array}{cc}
\mathbf{A} & \mathbf{O} \\
\mathbf{O} & \mathbf{D}
\end{array}\right)=\operatorname{det}_{F} \mathbf{A} \operatorname{det}_{F} \mathbf{D}
$$

The keen-eyed reader will notice immediately that, since

$$
\operatorname{det}_{F} \mathbf{A} \operatorname{det}_{F} \mathbf{D}=\operatorname{det}_{F}(\mathbf{A D}),
$$

equation (3) is just (2) in the special case where $\mathbf{B}=\mathbf{C}=\mathbf{O}$. However, we postpone this step, because with care we can obtain a proof of the multiplicative property (4) as a by-product of the main argument.

One way of proving (3) is to use the Laplace expansion of $\operatorname{det}_{F} \mathbf{M}$ by the first $n$ rows, which gives the result immediately. A more elementary proof runs thus: generalize to the case where $\mathbf{A}$ is $r \times r$ but $\mathbf{D}$ is still $n \times n$. The result is now obvious if $r=1$, by expanding by the first row. So use induction on $r$, expanding by the first row to perform the inductive step. (Details are left to the reader.) This result still holds if we know only that $\mathbf{B}=\mathbf{O}$, and the proof is exactly the same: we obtain

$$
\operatorname{det}_{F}\left(\begin{array}{cc}
\mathbf{A} & \mathbf{O} \\
\mathbf{C} & \mathbf{D}
\end{array}\right)=\operatorname{det}_{F} \mathbf{A} \operatorname{det}_{F} \mathbf{D}
$$

By taking transposes, or by repeating the proof using columns instead of rows, we also obtain the result when $\mathbf{C}=\mathbf{O}$, namely,

$$
\operatorname{det}_{F}\left(\begin{array}{cc}
\mathbf{A} & \mathbf{B} \\
\mathbf{O} & \mathbf{D}
\end{array}\right)=\operatorname{det}_{F} \mathbf{A} \operatorname{det}_{F} \mathbf{D}
$$


In order to prove (4) we need to assume something about determinants, and we shall assume that adding a multiple of one row (respectively, column) to another row (respectively, column) of a matrix does not alter its determinant. Since multiplying a matrix on the left (respectively, right) by a unitriangular matrix corresponds to performing a number of such operations on the rows (respectively, columns), it does not alter the determinant. (A unitriangular matrix is a triangular matrix with all diagonal entries equal to 1.) We shall also assume that $\operatorname{det}_{F} \mathbf{I}_{n}=1$, where $\mathbf{I}_{n}$ is the $n \times n$ identity matrix. So now observe that

$$
\left(\begin{array}{cc}
\mathbf{I}_{n} & -\mathbf{I}_{n} \\
\mathbf{O} & \mathbf{I}_{n}
\end{array}\right)\left(\begin{array}{cc}
\mathbf{I}_{n} & \mathbf{O} \\
\mathbf{I}_{n} & \mathbf{I}_{n}
\end{array}\right)\left(\begin{array}{cc}
\mathbf{I}_{n} & -\mathbf{I}_{n} \\
\mathbf{O} & \mathbf{I}_{n}
\end{array}\right)\left(\begin{array}{cc}
\mathbf{A} & \mathbf{B} \\
\mathbf{C} & \mathbf{D}
\end{array}\right)=\left(\begin{array}{cc}
-\mathbf{C} & -\mathbf{D} \\
\mathbf{A} & \mathbf{B}
\end{array}\right),
$$

whence $\operatorname{det}_{F}\left(\begin{array}{cc}\mathbf{A} & \mathbf{B} \\ \mathbf{C} & \mathbf{D}\end{array}\right)=\operatorname{det}_{F}\left(\begin{array}{cc}-\mathbf{C} & -\mathbf{D} \\ \mathbf{A} & \mathbf{B}\end{array}\right)$, since the first three matrices on the left of (7) are unitriangular. From (5) and (6) it follows from this that

$$
\operatorname{det}_{F}\left(\begin{array}{cc}
\mathbf{A} & \mathbf{B} \\
\mathbf{C} & \mathbf{O}
\end{array}\right)=\operatorname{det}_{F}(-\mathbf{C}) \operatorname{det}_{F} \mathbf{B}=\operatorname{det}_{F}\left(\begin{array}{cc}
\mathbf{O} & \mathbf{B} \\
\mathbf{C} & \mathbf{D}
\end{array}\right)
$$

But also

$$
\left(\begin{array}{cc}
\mathbf{A} & \mathbf{O} \\
-\mathbf{I}_{n} & \mathbf{D}
\end{array}\right)\left(\begin{array}{cc}
\mathbf{I}_{n} & \mathbf{D} \\
\mathbf{O} & \mathbf{I}_{n}
\end{array}\right)=\left(\begin{array}{cc}
\mathbf{A} & \mathbf{A D} \\
-\mathbf{I}_{n} & \mathrm{O}
\end{array}\right)
$$

Here the second matrix on the left is unitriangular, so taking determinants and using (5) and the first part of (8), we have

$$
\operatorname{det}_{F} \mathbf{A} \operatorname{det}_{F} \mathbf{D}=\operatorname{det}_{F} \mathbf{I}_{n} \operatorname{det}_{F}(\mathbf{A D})
$$

and since $\operatorname{det}_{F} \mathbf{I}_{n}=1$, the multiplicative law (4) for determinants in ${ }^{n} F^{n}$ follows.

\section{Determinants of $2 \times 2$ block matrices}

Since we now know that $\operatorname{det}_{F} \mathbf{A} \operatorname{det}_{F} \mathbf{D}=\operatorname{det}_{F}(\mathbf{A D})$, then $\operatorname{also} \operatorname{det}_{F}(-\mathbf{C}) \operatorname{det}_{F} \mathbf{B}=$ $\operatorname{det}_{F} \mathbf{B} \operatorname{det}_{F}(-\mathbf{C})=\operatorname{det}_{F}(\mathbf{B}(-\mathbf{C}))=\operatorname{det}_{F}(-\mathbf{B C})$. From (5), (6) and (8), we obtain:

Lemma 2. If $\mathbf{M}=\left(\begin{array}{cc}\mathbf{A} & \mathbf{B} \\ \mathbf{C} & \mathbf{D}\end{array}\right)$, then

$$
\operatorname{det}_{F} \mathbf{M}=\operatorname{det}_{F}(\mathbf{A D}-\mathbf{B C})
$$

whenever at least one of the blocks $\mathbf{A}, \mathbf{B}, \mathbf{C}, \mathbf{D}$ is equal to $\mathbf{O}$.

(Compare this with (2).)

We shall now try to generalize somewhat. Suppose the blocks $\mathbf{C}$ and $\mathbf{D}$ commute, that is, $\mathbf{C D}=\mathbf{D C}$. Then

$$
\left(\begin{array}{cc}
\mathrm{A} & \mathrm{B} \\
\mathrm{C} & \mathrm{D}
\end{array}\right)\left(\begin{array}{cc}
\mathrm{D} & \mathrm{O} \\
-\mathrm{C} & \mathrm{I}_{n}
\end{array}\right)=\left(\begin{array}{cc}
\mathrm{AD}-\mathrm{BC} & \mathrm{B} \\
\mathrm{CD}-\mathrm{DC} & \mathrm{D}
\end{array}\right)=\left(\begin{array}{cc}
\mathrm{AD}-\mathrm{BC} & \mathrm{B} \\
\mathrm{O} & \mathrm{D}
\end{array}\right) .
$$


We proved (4) in ${ }^{n} F^{n}$ for all $n$, so we can apply it in ${ }^{2 n} F^{2 n}$ to get, via (5) and (6),

$$
\operatorname{det}_{F} \mathbf{M} \operatorname{det}_{F} \mathbf{D}=\operatorname{det}_{F}(\mathbf{A D}-\mathbf{B C}) \operatorname{det}_{F} \mathbf{D},
$$

and so

$$
\left(\operatorname{det}_{F} \mathbf{M}-\operatorname{det}_{F}(\mathbf{A D}-\mathbf{B C})\right) \operatorname{det}_{F} \mathbf{D}=0 .
$$

Now if $\operatorname{det}_{F} \mathbf{D}$ is not zero (or, in the case where $F$ is a ring rather than a field, if $\operatorname{det}_{F} \mathbf{D}$ is not a divisor of zero), then (9) follows immediately from (11); but we do not actually need this extra assumption, as we shall now show. Adjoin an indeterminate $x$ to $F$, and work in the polynomial ring $F[x]$. This is a(nother) commutative ring, and a typical element is a polynomial $a_{0} x^{r}+a_{1} x^{r-1}+\ldots+a_{r-1} x+a_{r}$, where $a_{i} \in F$, all $i$, and addition, subtraction and multiplication of polynomials is done in the obvious way. Now let us add to $\mathbf{D}$ the scalar matrix $x \mathbf{I}_{n}$, and for brevity write $\mathbf{D}_{x}=x \mathbf{I}_{n}+\mathbf{D}$. Since a scalar matrix necessarily commutes with $\mathbf{C}$ (because it commutes with every matrix), and also $\mathbf{D}$ commutes with $\mathbf{C}$, it follows that $\mathbf{D}_{x}$ commutes with $\mathbf{C}$. Thus, if we put $\mathbf{M}_{x}=\left(\begin{array}{cc}\mathbf{A} & \mathbf{B} \\ \mathbf{C} & \mathbf{D}_{x}\end{array}\right)$, then working over $F[x]$ in place of $F$ yields equation (11) for $\mathbf{M}_{x}$, that is,

$$
\left(\operatorname{det}_{F} \mathbf{M}_{x}-\operatorname{det}_{F}\left(\mathbf{A D}_{x}-\mathbf{B C}\right)\right) \operatorname{det}_{F} \mathbf{D}_{x}=0 .
$$

But here we have the product of two polynomials equal to the zero polynomial. The second polynomial $\operatorname{det}_{F} \mathbf{D}_{x}=\operatorname{det}_{F}\left(x \mathbf{I}_{n}+\mathbf{D}\right)$ is certainly not the zero polynomial, but is monic of degree $n$, that is, it is of the form $x^{n}+$ terms of lower degree. (It is in fact the characteristic polynomial of $-\mathbf{D}$.) This means that the left-hand polynomial in (12), the bracketed expression, must be the zero polynomial. For if not, it is of the form $a_{0} x^{r}+$ terms of lower degree, where $a_{0} \in F$ and $a_{0} \neq 0$. Multiplying the two polynomials, we get $a_{0} x^{r+n}+$ terms of lower degree, which cannot be the zero polynomial, a contradiction. (This argument works even when $F$ is not a field, but merely a commutative ring possibly with divisors of zero.) So now we have proved that $\operatorname{det}_{F} \mathbf{M}_{x}-\operatorname{det}_{F}\left(\mathbf{A D}_{x}-\mathbf{B C}\right)=0$; but $\mathbf{D}_{0}=\mathbf{D}$ and $\mathbf{M}_{0}=\mathbf{M}$, so we just have to put $x=0$ and we obtain the following stronger version of Theorem 1:

$$
\begin{aligned}
& \text { Theorem 3. If } \mathbf{M}=\left(\begin{array}{cc}
\mathbf{A} & \mathbf{B} \\
\mathbf{C} & \mathbf{D}
\end{array}\right) \text {, where } \mathbf{A}, \mathbf{B}, \mathbf{C}, \mathbf{D} \in{ }^{n} F^{n} \text { and } \mathbf{C D}=\mathbf{D C} \text {, then } \\
& \operatorname{det}_{F} \mathbf{M}=\operatorname{det}_{F}(\mathbf{A D}-\mathbf{B C}) .
\end{aligned}
$$

Other versions of Theorem 3 hold if different blocks of $\mathbf{M}$ commute, so it is an exercise for the reader to show that

$$
\begin{aligned}
& \text { if } \mathbf{A C}=\mathbf{C A} \text { then } \operatorname{det}_{F} \mathbf{M}=\operatorname{det}_{F}(\mathbf{A D}-\mathbf{C B}) \text {; } \\
& \text { if } \mathbf{B D}=\mathbf{D B} \text { then } \operatorname{det}_{F} \mathbf{M}=\operatorname{det}_{F}(\mathbf{D A}-\mathbf{B C}) \text {; } \\
& \text { if } \mathbf{A B}=\mathbf{B A} \text { then } \operatorname{det}_{F} \mathbf{M}=\operatorname{det}_{F}(\mathbf{D A}-\mathbf{C B}) \text {. }
\end{aligned}
$$

Of course, if all four blocks of $\mathbf{M}$ commute pairwise, then all of (13), (14), (15), (16) hold, the expressions on the right being equal. As a particular case, if $R$ is a commutative subring of ${ }^{n} F^{n}$ and $\mathbf{A}, \mathbf{B}, \mathbf{C}, \mathbf{D} \in R$, then any of (13)-(16) gives an expression for $\operatorname{det}_{F} \mathbf{M}$, and this gives a proof of Theorem 1 in the case $m=2$. 


\section{Determinants of $\mathrm{m} \times \mathrm{m}$ block matrices}

In this section we shall prove Theorem 1, but first we do some preliminary calculations. Let $R$ be any commutative ring, and let $\mathbf{M} \in{ }^{m} R^{m}$, where $m \geq 2$. We partition $\mathbf{M}$ as

follows: $\mathbf{M}=\left(\begin{array}{cc}\mathbf{A} & \mathbf{b} \\ \mathbf{c} & d\end{array}\right)$, where $\mathbf{A} \in{ }^{m-1} R^{m-1}, \mathbf{b} \in{ }^{m-1} R, \mathbf{c} \in R^{m-1}$, and $d \in R$. So here $\mathbf{b}$ is a column vector, and $\mathbf{c}$ is a row vector. Suppose $\mathbf{c}=\left(c_{1}, c_{2}, \ldots, c_{m-1}\right)$, and consider the effect of multiplying this on the right by the scalar matrix $d \mathbf{I}_{m-1}$. We have

$$
\begin{aligned}
\mathbf{c}\left(d \mathbf{I}_{m-1}\right) & =\left(c_{1}, c_{2}, \ldots, c_{m-1}\right)\left(\begin{array}{cccc}
d & 0 & \ldots & 0 \\
0 & d & \ldots & 0 \\
\vdots & \vdots & \ddots & \vdots \\
0 & 0 & \ldots & d
\end{array}\right) \\
& =\left(c_{1} d, c_{2} d, \ldots, c_{m-1} d\right) \\
& =\left(d c_{1}, d c_{2}, \ldots, d c_{m-1}\right) \quad \text { (because } R \text { is commutative) } \\
& =d\left(c_{1}, c_{2}, \ldots, c_{m-1}\right)=d \mathbf{c} .
\end{aligned}
$$

In a similar way, $\mathbf{A}\left(d \mathbf{I}_{m-1}\right)=d \mathbf{A}$. So now we have

$$
\left(\begin{array}{cc}
\mathbf{A} & \mathbf{b} \\
\mathbf{c} & d
\end{array}\right)\left(\begin{array}{cc}
d \mathbf{I}_{m-1} & \mathbf{0} \\
-\mathbf{c} & 1
\end{array}\right)=\left(\begin{array}{cc}
\mathbf{A}_{0} & \mathbf{b} \\
\mathbf{0} & d
\end{array}\right)
$$

where $\mathbf{A}_{0}=d \mathbf{A}-\mathbf{b} \mathbf{c} \in{ }^{m-1} R^{m-1}$. (Notice here that $\mathbf{b c}$, being the product of an $(m-1) \times 1$ matrix and a $1 \times(m-1)$ matrix, is an $(m-1) \times(m-1)$ matrix.) Applying $\operatorname{det}_{R}$ to each side of (17), we get

$$
\left(\operatorname{det}_{R} \mathbf{M}\right) d^{m-1}=\left(\operatorname{det}_{R} \mathbf{A}_{0}\right) d .
$$

If we now take $R$ to be a commutative subring of ${ }^{n} F^{n}$, then (18) is an equation in ${ }^{n} F^{n}$, so we can apply $\operatorname{det}_{F}$ to each side and get

$$
\operatorname{det}_{F}\left(\operatorname{det}_{R} \mathbf{M}\right)\left(\operatorname{det}_{F} d\right)^{m-1}=\operatorname{det}_{F}\left(\operatorname{det}_{R} \mathbf{A}_{0}\right)\left(\operatorname{det}_{F} d\right)
$$

Also, (17) is now an equation in ${ }^{m n} F^{m n}$, so we can apply $\operatorname{det}_{F}$ to each side and obtain

$$
\left(\operatorname{det}_{F} \mathbf{M}\right)\left(\operatorname{det}_{F} d\right)^{m-1}=\left(\operatorname{det}_{F} \mathbf{A}_{0}\right)\left(\operatorname{det}_{F} d\right) .
$$

We now have everything we need ready for a proof of Theorem 1 .

Proof of Theorem 1. The result is trivial if $m=1$, for then $\operatorname{det}_{R} \mathbf{M}=\mathbf{M}$, and there is nothing to prove. So assume $m \geq 2$, partition $\mathbf{M}$ as above, and use induction on $m$. By the inductive hypothesis, $\operatorname{det}_{F}\left(\operatorname{det}_{R} \mathbf{A}_{0}\right)=\operatorname{det}_{F} \mathbf{A}_{0}$, since $\mathbf{A}_{0} \in{ }^{m-1} R^{m-1}$. From (19) and (20) we deduce

$$
\left(\operatorname{det}_{F} \mathbf{M}-\operatorname{det}_{F}\left(\operatorname{det}_{R} \mathbf{M}\right)\right)\left(\operatorname{det}_{F} d\right)^{m-1}=0 .
$$

Now if $\operatorname{det}_{F} d$ is not zero (or, in the case where $F$ is a commutative ring rather than a field, if $\operatorname{det}_{F} d$ is not a divisor of zero), then our result follows immediately. To deal with other cases, we repeat our trick with polynomials in the proof of Lemma 2, in section 3. 
Adjoin an indeterminate $x$ to $F$, and work in the polynomial ring $F[x]$. Add the scalar matrix $x \mathbf{I}_{n}$ to $d$ to define $d_{x}=x \mathbf{I}_{n}+d$ (which may look strange, but $d$, being in $R$, really is an $n \times n$ matrix over $F$ ), and make the corresponding adjustment to $\mathbf{M}$, by putting $\mathbf{M}_{x}=\left(\begin{array}{cc}\mathbf{A} & \mathbf{b} \\ \mathbf{c} & d_{x}\end{array}\right)$. Then exactly as for $\mathbf{M}$, we have

$$
\left(\operatorname{det}_{F} \mathbf{M}_{x}-\operatorname{det}_{F}\left(\operatorname{det}_{R} \mathbf{M}_{x}\right)\right)\left(\operatorname{det}_{F} d_{x}\right)^{m-1}=0 .
$$

But $\operatorname{det}_{F} d_{x}$ is a monic polynomial of degree $n$, and we conclude that the other term in (22), $\operatorname{det}_{F} \mathbf{M}_{x}-\operatorname{det}_{F}\left(\operatorname{det}_{R} \mathbf{M}_{x}\right)$, is the zero polynomial. Putting $x=0$ and noting that $d_{0}=d$ and $\mathbf{M}_{0}=\mathbf{M}$, we obtain $\operatorname{det}_{F} \mathbf{M}=\operatorname{det}_{F}\left(\operatorname{det}_{R} \mathbf{M}\right)$, and the proof of Theorem 1 is complete.

As an application, we can obtain the formula for the determinant of a tensor product of matrices rather easily. For example, if $\mathbf{P}=\left(\begin{array}{ll}p_{11} & p_{12} \\ p_{21} & p_{22}\end{array}\right) \in{ }^{2} F^{2}$ and $\mathbf{Q} \in{ }^{n} F^{n}$, the tensor product $\mathbf{P} \otimes \mathbf{Q}$ is defined to be the $2 n \times 2 n$ matrix $\left(\begin{array}{ll}p_{11} \mathbf{Q} & p_{12} \mathbf{Q} \\ p_{21} \mathbf{Q} & p_{22} \mathbf{Q}\end{array}\right)$. Here the blocks, all being scalar multiples of $\mathbf{Q}$, commute pairwise; one could in fact take $R$ to be the subring of ${ }^{n} F^{n}$ generated by $\mathbf{Q}$, that is, the set $F[\mathbf{Q}]$ of all polynomial expressions $a_{0} \mathbf{Q}^{r}+a_{1} \mathbf{Q}^{r-1}+\ldots+a_{r-1} \mathbf{Q}+a_{r} \mathbf{I}_{n}$, where $r \geq 0$ and $a_{i} \in F$, all $i$. This is certainly a commutative subring of ${ }^{n} F^{n}$ and contains all four blocks $p_{i j} \mathbf{Q}$. Applying Theorem 1, or Theorem 3, we have

$$
\begin{aligned}
\operatorname{det}_{F}(\mathbf{P} \otimes \mathbf{Q}) & =\operatorname{det}_{F}\left(\left(p_{11} \mathbf{Q}\right)\left(p_{22} \mathbf{Q}\right)-\left(p_{12} \mathbf{Q}\right)\left(p_{21} \mathbf{Q}\right)\right) \\
& =\operatorname{det}_{F}\left(p_{11} p_{22} \mathbf{Q}^{2}-p_{12} p_{21} \mathbf{Q}^{2}\right) \\
& =\operatorname{det}_{F}\left(\left(p_{11} p_{22}-p_{12} p_{21}\right) \mathbf{Q}^{2}\right) \\
& =\operatorname{det}_{F}\left(\left(\operatorname{det}_{F} \mathbf{P}\right) \mathbf{Q}^{2}\right) \\
& =\left(\operatorname{det}_{F} \mathbf{P}\right)^{n}\left(\operatorname{det}_{F} \mathbf{Q}\right)^{2}
\end{aligned}
$$

More generally:

Corollary. Let $\mathbf{P} \in{ }^{m} F^{m}$ and $\mathbf{Q} \in{ }^{n} F^{n}$. Then

$$
\operatorname{det}_{F}(\mathbf{P} \otimes \mathbf{Q})=\left(\operatorname{det}_{F} \mathbf{P}\right)^{n}\left(\operatorname{det}_{F} \mathbf{Q}\right)^{m}
$$

Proof. Take $R=F[\mathbf{Q}]$, as above. If $\mathbf{P}=\left(p_{i j}\right)$, then by definition

$$
\mathbf{P} \otimes \mathbf{Q}=\left(\begin{array}{cccc}
p_{11} \mathbf{Q} & p_{12} \mathbf{Q} & \ldots & p_{1 m} \mathbf{Q} \\
p_{21} \mathbf{Q} & p_{22} \mathbf{Q} & \ldots & p_{2 m} \mathbf{Q} \\
\vdots & \vdots & \ddots & \vdots \\
p_{m 1} \mathbf{Q} & p_{m 2} \mathbf{Q} & \ldots & p_{m m} \mathbf{Q}
\end{array}\right) \in{ }^{m} R^{m}
$$

Then $\operatorname{det}_{R}(\mathbf{P} \otimes \mathbf{Q})$ is a sum of $m$ ! terms each of the form

$$
\pm\left(p_{1 i_{1}} \mathbf{Q}\right)\left(p_{2 i_{2}} \mathbf{Q}\right) \ldots\left(p_{m i_{m}} \mathbf{Q}\right)= \pm p_{1 i_{1}} p_{2 i_{2}} \ldots p_{m i_{m}} \mathbf{Q}^{m}
$$


for some permutation $i_{1}, i_{2}, \ldots, i_{m}$ of $1,2, \ldots, m$. Thus

$$
\operatorname{det}_{R}(\mathbf{P} \otimes \mathbf{Q})=\left(\operatorname{det}_{F} \mathbf{P}\right) \mathbf{Q}^{m}
$$

Then, by Theorem 1,

$$
\begin{aligned}
\operatorname{det}_{F}(\mathbf{P} \otimes \mathbf{Q}) & =\operatorname{det}_{F}\left(\operatorname{det}_{R}(\mathbf{P} \otimes \mathbf{Q})\right) \\
& =\operatorname{det}_{F}\left(\left(\operatorname{det}_{F} \mathbf{P}\right) \mathbf{Q}^{m}\right) \\
& =\left(\operatorname{det}_{F} \mathbf{P}\right)^{n}\left(\operatorname{det}_{F} \mathbf{Q}^{m}\right) \\
& =\left(\operatorname{det}_{F} \mathbf{P}\right)^{n}\left(\operatorname{det}_{F} \mathbf{Q}\right)^{m}
\end{aligned}
$$

\section{Acknowledgements}

This paper began when I set my linear algebra class the following exercise: if $\mathbf{A}, \mathbf{B}, \mathbf{C}$, $\mathbf{D} \in{ }^{n} F^{n}$ and $\mathbf{D}$ is invertible, show that

$$
\operatorname{det}\left(\begin{array}{ll}
\mathbf{A} & \mathbf{B} \\
\mathbf{C} & \mathbf{D}
\end{array}\right)=\operatorname{det}\left(\mathbf{A D}-\mathbf{B D}^{-1} \mathbf{C D}\right)
$$

(The intended proof was via the equation

$$
\left(\begin{array}{ll}
\mathbf{A} & \mathbf{B} \\
\mathbf{C} & \mathbf{D}
\end{array}\right)\left(\begin{array}{cc}
\mathbf{I}_{n} & \mathbf{O} \\
-\mathbf{D}^{-1} \mathbf{C} & \mathbf{I}_{n}
\end{array}\right)=\left(\begin{array}{cc}
\mathbf{A}-\mathbf{B D}^{-1} \mathbf{C} & \mathbf{B} \\
\mathbf{O} & \mathbf{D}
\end{array}\right)
$$

and the exercise took its inspiration from the theory of Dieudonné determinants for matrices over skew fields. See, for example, [1], chapter IV.) It was a small step to notice that, if also $\mathbf{C D}=\mathbf{D C}$, then (13) holds; but (13) does not mention $\mathbf{D}^{-1}$, and so the natural question to ask was whether (13) would hold even if $\mathbf{D}$ were not invertible (but still $\mathbf{C D}=\mathbf{D C}$ ). I eventually found a proof of this, when $F$ is a field, by using the principle of the irrelevance of algebraic inequalities. (See [3], chapter 6.) I passed this around friends and colleagues, and Dr W. Stephenson showed me how to use monic polynomials to shorten the argument, and extend it from fields to rings. At the same time, Dr A. D. Barnard suggested it might be possible to extend the result to the $m \times m$ case by assuming all the pairs of blocks commute. I am grateful to both of them for their help, and their interest. I also wish to thank the referee for directing my attention to some of the early literature on this subject, mentioned below.

Theorem 1 is not, I think, a new result, and I have seen what appears to be an abstract version of it (without proof) at a much more advanced level. I have not been able to find an elementary statement and proof, as given above, in the literature. The block matrix proof of the multiplicative property of determinants is essentially that given in [2], chapter 4. The formula for the determinant of a tensor product first appears in the case $m=4, n=2$ in [11], and indeed is referred to in [7] as Zehfuss' theorem. The first proof of the general case is probably that in [8], p. 117, though in [5], p. 82, the proof is attributed to [4], [9] and [10]. See also the references to these articles in [7], volume 2, pp. 102-104, and volume 4, pp. 42, 62 and 216. 


\section{References}

[1] E. Artin, Geometric Algebra, Interscience 1957.

[2] Frank Ayres, Jr., Matrices, Shaum's Outline Series, McGraw-Hill 1962.

[3] P. M. Cohn, Algebra, Volume 1, Wiley 1982 (2nd edition).

[4] K. Hensel, Über die Darstellung der Determinante eines Systems welches aus zwei anderen componirt ist, Acta Math. 14 (1889), 317-319.

[5] C. C. MacDuffee, The theory of matrices, Chelsea 1956.

[6] L. Mirsky, An introduction to linear algebra, Clarendon Press 1955.

[7] T. Muir, The theory of determinants in the historical order of development, Dover reprint 1960 .

[8] T. Muir, A treatise on determinants, Macmillan 1881.

[9] E. Netto, Zwei Determinantensätze, Acta Math. 17 (1893), 199-204.

[10] R. D. von Sterneck, Beweis eines Satzes über Determinanten, Monatshefte f. Math. u. Phys. 6 (1895), 205-207.

[11] G. Zehfuss, Über eine gewisse Determinante, Zeitschrift f. Math. u. Phys. 3 (1858), 298-301.

Department of Mathematics

7 September 1999

King's College

Strand

London WC2R 2LS

(jrs@kcl.ac.uk) 